\title{
Automated Segmentation of Cardiac Stenosis and Mathematical Modeling of Myocardial Blood Flow for Early Detection of Heart Attack Using Advanced Imaging Techniques
}

\author{
Supriya Agrawal \\ (Assistant professor, Department of Computer Science, Mukesh Patel School of Technology Management \& \\ Engineering, NMIMS University, Mumbai)
}

\begin{abstract}
Heart attack is a leading cause of human deaths worldwide which occurs due to occlusion of coronary arteries and reduced blood flow. Hence, it requires early detection of disease in a non-invasive way through Cardiac Computed Tomography Angiogram images (CCTA). Local thresholding with Hessian matrix based Frangi's Vesselness filter has been applied for segmentation of coronary arterial branches. Stenosis as Region of Interest can be quantified in cardiac arteries by using Sobel Gradient Edge operator with threshold values 76 to 80. Mathematical model has been designed to measure the rate of change of blood flow in coronary arteries by adopting hemodynamic fluid mechanics using Hagen Poiseuille's law with Wall Shear Stress method. Computerized simulation results assist to detect healthy and cardiac diseased artery for better diagnosis.
\end{abstract}

Keywords - Coronary Artery Disease, Heart Attack, Blood Flow, CT Angiogram, Fluid Mechanics

\section{Introduction}

According to recent statistics of World Health Organization (WHO), almost 17.3 million people die due to Coronary Artery Disease (CAD) every year which is $30.2 \%$ of all deaths. Today, health care systems deeply rely on medical information system by extracting knowledge of medical images with advances of digital image processing. Coronary artery disease leads to blockage inside the cardiac artery due to waxy material called plaque which results to heart attack. The objective of this research work is to identify the risk factors of heart attack or myocardial infarction at early stages in non-invasive manner for better surgical treatment. In this context, segmentation techniques with vessel filter have been applied on CT angiogram images to segment region of interest (ROI) such as coronary arterial branches and degree of stenosis or blockage inside the coronary artery. The proposed mathematical model describes the rate of change of blood flow through cardiac vessels by using Hagen Poiseuille [5] fluid equation and Wall Shear Stress method. This paper is further divided as follows: section (2) describes research methodology; section (3) covers computer simulation results and section (4) conclusion of the study.

\section{Research Methodology}

This work proposes the following successive steps for early prediction of heart attack:

(i) Extraction of coronary arteries

(ii) Detection of stenosis in segmented arteries

(iii) Calculate cross sectional area of healthy and diseased cardiac arteries

(iv) Designing a mathematical model for measuring rate of change of blood flow through healthy and diseased coronary vessels

\subsection{Data Acquisition}

The acquired datasets are real Cardiac Computed Tomography Angiography (CCTA) images in DICOM format taken from Dr. Balabhai Nanavati Hospital, Mumbai and Cardiac Life Center, Ludhiana. Experiments have been done on small batches of normal as well as patients' data of both males (age group 40 to $65 \mathrm{yrs}$ ) and females (age group 35to55 yrs).

\subsection{Pre-Processing for Image Analysis}

\subsubsection{Image Enhancement \& Pre-Segmentation}

The aim of image enhancement technique is to improve image quality for better visual perception. In this study, median filter has been applied on gray label CCTA images followed by local threshold techniques with morphological operator as shown in "Fig (1-d)". 


\subsubsection{Segmenting Coronary Arterial Branches}

Segmentation of coronary arterial tree is a critical component in image processing because cardiac arteries are in tubular form thereby requiring computer systems. In this work, coronary arteries are taken as Region of Interest (ROI) and to extract the structure of coronary arteries from CCTA medical images, we have used Hassian Matrix based Frangi's vesselness filter as shown in "Fig(1-e)".

Hassian matrix is the square matrix of second order partial derivative which describes local curvature of the image. For a given voxel of input image, the eigenvalue of Hessian matrix is oriented on the basis of vesselness filter $[1,8]$.

$$
\mathrm{H}=\left[\begin{array}{ccc}
\partial^{2} I / \partial x^{2} & \partial^{2} I / \partial x \partial y & \partial^{2} I / \partial x \partial z \\
\partial^{2} I / \partial x \partial y & \partial^{2} I / \partial y^{2} & \partial^{2} I / \partial y \partial z \\
\partial^{2} I / \partial x \partial z & \partial^{2} I / \partial z \partial y & \partial^{2} I / \partial z^{2}
\end{array}\right]
$$

where I refers to the input image and $\partial$ refer to derivatives of the image. In our approach we have used vessel enhancement as a filtering process that searches for geometrical structure in a second order directional derivative [8]. Vessel structure is brighter than background and Eigen Values $\lambda$ calculate grey-label invariants by following dissimilarity measure ratios $[1,8]$

$$
R_{A}=\frac{\left|\lambda_{2}\right|}{\left|\lambda_{3}\right|} \quad \text { and } \quad R_{B}=\frac{\left|\lambda_{1}\right|}{\sqrt{\left|\lambda_{2} \lambda_{3}\right|}}
$$

We can calculate vessel values of "second order structureness" by proposed vesselness function

$$
\begin{aligned}
& \mathrm{S}=\|H\|_{F}=\sqrt{\sum_{i<3} \lambda_{i}}{ }^{2}, \quad \text { if } \lambda_{2}>0 \text { or } \lambda_{3}>0 \\
& v_{0}(s)=\left\{\begin{array}{cc}
0, \\
\left(1-\exp \left(-\frac{R_{A}{ }^{2}}{2 \alpha^{2}}\right)\right) \exp \left(-\frac{R_{\rho}{ }^{2}}{2 \beta^{2}}\right)\left(1-\exp \left(-\frac{s^{2}}{2 \sigma^{2}}\right)\right)
\end{array}\right.
\end{aligned}
$$

where $\alpha_{3} \beta$ and c are threshold values to measure $R_{A} R_{B}$ and $\mathrm{S}$. The idea behind this calculation is to estimate vessel structure according to different criteria [8].

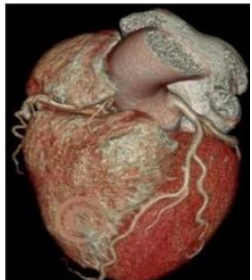

(a)

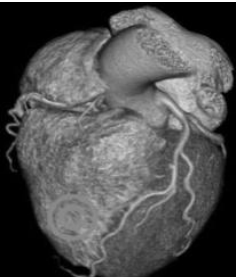

(b)

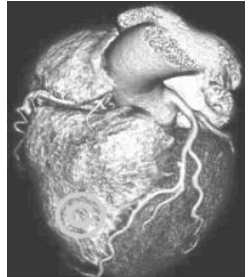

(c)

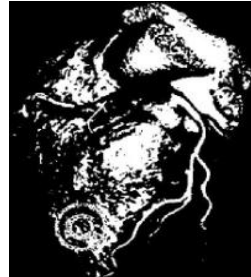

(d)

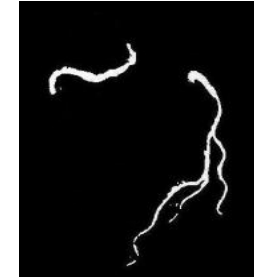

(e)

Fig.1: Sequences of Coronary Artery Tree Extraction Algorithm.

(a) Original CT Image of 60 yr Old Man, (b) Grey Image, (c) Enhanced Image, (d) Image after Thresholding and Morphology Operator, (e) Vessel Filter Image

\subsection{Coronary Stenosis Detection}

Stenosis is a condition in which coronary arteries become narrow due to which blood flow is reduced inside the heart. To detect any abnormality in segmented arterial branches, Sobel Gradient Edge Operator with threshold value 76 and 80 has been applied for performing spatial gradient measurement and emphasizing regions of high spatial frequency that correspond to edges as shown in "Fig(2 3)". At each point in the image, the resulting gradient magnitude is computed using the formula

$$
|G|=\sqrt{G x^{2}+G y^{2}}
$$

An approximate value can be calculated using

$$
|G|=|G x|+|G y|
$$




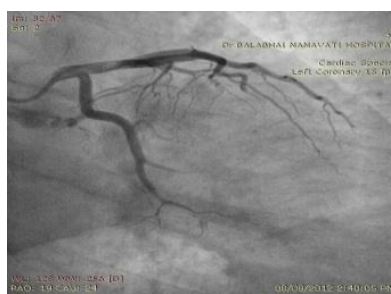

(a)

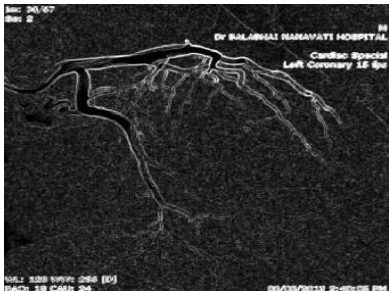

(b)

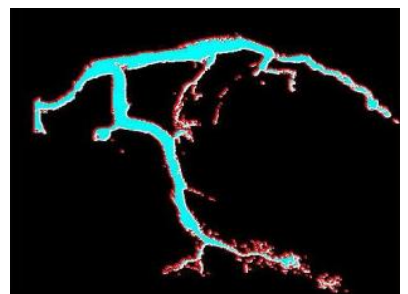

( c )

Fig.2: (a) CTA of Healthy Left Coronary Branches, (b) Sobel Edge Gradient Image, (c) Image after Segmentation Process and Threshold Value 76

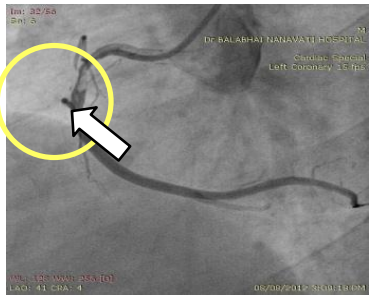

(a) 55 yr male patient angiogram image

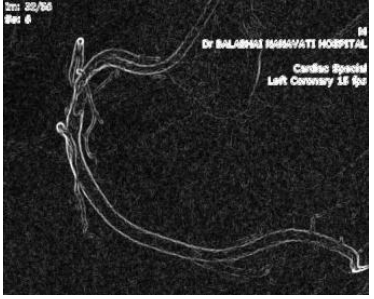

(b) Image after Sobel edg $\epsilon$ gradient operation

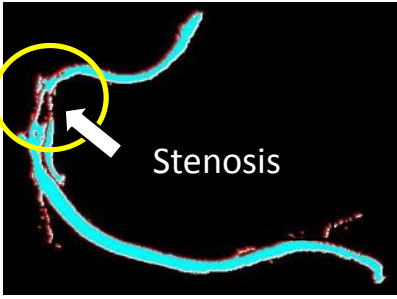

(c) Image after segmentation process with threshold value 80 to detect stenosis

\subsection{Calculate Cross Sectional Area by Ellipse Fitting Method}

The next step is to calculate cross sectional area of segmented arteries for differentiating healthy and diseased cardiac vessels. We used Ellipse curve fitting method [9] as second order central moment mentioned in below equation

$$
\frac{1}{1+\sum_{i, j=0}^{i+j<=4}\left(\mu_{i j}^{\prime}-\mu_{i j}\right)^{2}}
$$

where $\boldsymbol{\mu}_{i j}$ and $\boldsymbol{\mu}_{i j}$ are central moments of the shape and corresponding ellipse fit. Then we calculated the area of curve fitting by Eq. 8 as result shown in under Section 3.3 (Table 3)

$$
A_{0}=\pi \times r_{1} \times r_{2}
$$

where $\boldsymbol{r}_{1}$ is semi major axis and $\boldsymbol{r}_{2}$ is semi minor axis.

(a)

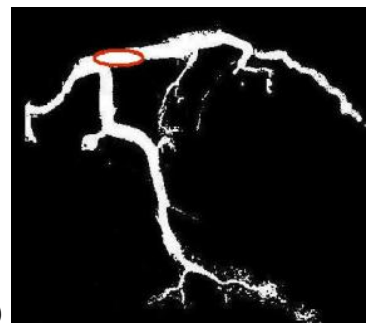

(b)

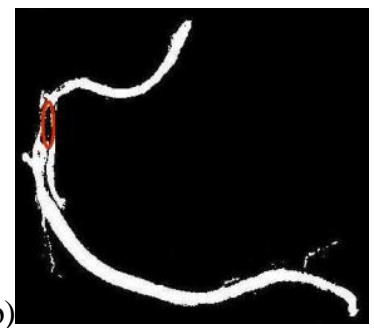

Fig.4: (a) (b) Represents Ellipse Fitting method on Healthy and Diseased Coronary Arteries

\subsection{Mathematical Modeling of Blood Flow}

As per a clinical study, normal blood pressure is $120 / 80 \mathrm{mmhg}$ in males and $110 / 70 \mathrm{mmhg}$ in females. If blood pressure is very high from the normal pressure then coronary heart disease (CHD) may arises. The next focus of this work is designing a mathematical model to quantify the rate of change of blood flow in segmented cardiac vessels for early prediction of heart attack which can take place in any age of both males and females.

Blood is a non-Newtonian fluid and to model such liquid motion equations becomes complicated so we have assumed that blood is incompressible Newtonian fluid. Coronary artery flow is strongly influenced by contraction and relaxation of ventricles and atria [3]. This study also follows the viscoelastic model of Skalak and Schmid-Schonbein [5] in which pressure changes in linear form related to strain, such that

$$
\Delta P=\alpha E
$$

where $\Delta P=P_{A}-P_{V} ;$ pressure difference of Atria and Ventricles and Strain $\mathrm{E}$ as defined as 


$$
E=\frac{1}{2}\left\{\left[\frac{a(z, t)}{a_{0}}\right]^{2}-1\right\}
$$

where $\alpha$ is elasticity constant, $a_{0}$ is reference radius and a $(\mathrm{z}, \mathrm{t})$ is radius of coronary artery. Coronary length is vary according to male and female and length changes is expressed by

$$
\ell(t)=\omega(t) \ell_{0}
$$

where $\ell(t)$ is time dependent length, $\omega(t)$ is relative length change of adjacent heart muscles.

Now, blood flow, non homogeneous non-Newtonian fluid, is dependent on vessel diameter and resistance in cardiovascular system as explained in Poiseuille's law

$$
Q=-\frac{\pi a^{4}}{8 \mu \ell} \Delta P
$$

where $\ell$ represents length of artery, $\mu$ represents blood viscosity and $\mathrm{Q}$ represents blood flow.

The relation between resistance and cardiac vessel diameter is inversely proportional as given below

$$
R=\frac{P_{A}-P_{B}}{Q}=\frac{8 \mu \ell}{\pi r^{4}}
$$

The size of coronary arteries can be deformed due to considered cross sectional plane, and blood flow inside the artery's wall that produces stress [3] as given below

$$
\text { Wall Shear Stress }=\mu \frac{\delta v}{\delta r}
$$

Poiseuille law can be applied to find out shear rate $\gamma$ as

$$
\gamma=\frac{8 \mu}{d} \quad \text { or } \quad \gamma=\frac{32 \mu}{\pi d^{3}} ; \text { where } d \text { is the diameter of artery }
$$

\section{Results And Discussions}

We have evaluated a mathematical model for cardiac blood flow system which includes patients as well as healthy human specific factors. Computerized simulations have been implemented on MATLAB 7.9(R2009B) software.

\subsection{Clinical Study}

This research has been done on small batches of healthy as well as diseased Left Coronary Artery (LCA) and Right Coronary Artery (RCA) angiogram images. Simulation of blood flow rate based on clinically

\begin{tabular}{|c|c|c|c|c|c|c|c|c|}
\hline \multirow{2}{*}{$\begin{array}{l}\text { Coronary } \\
\text { Arteries }\end{array}$} & \multirow{2}{*}{ Sex } & \multirow{2}{*}{$\begin{array}{l}\text { Length ( } \\
\mathrm{mm})\end{array}$} & \multicolumn{2}{|c|}{ Diameter ( mm ) } & \multicolumn{2}{|l|}{ Resistance } & \multicolumn{2}{|c|}{$\begin{array}{l}\text { Rate of Change of Blood } \\
\text { Flow }(\mathrm{ml} / \mathrm{sec})\end{array}$} \\
\hline & & & $\begin{array}{l}\text { Healthy } \\
\text { Coronary }\end{array}$ & $\begin{array}{l}\text { Diseased } \\
\text { Coronary }\end{array}$ & $\begin{array}{l}\text { Healthy } \\
\text { Coronary }\end{array}$ & $\begin{array}{l}\text { Diseased } \\
\text { Coronary }\end{array}$ & $\begin{array}{l}\text { Healthy } \\
\text { Coronary }\end{array}$ & $\begin{array}{l}\text { Diseased } \\
\text { Coronary }\end{array}$ \\
\hline LCA & $\mathrm{M}$ & 9.6 & 4.6 & 3.8 & 0.0012 & 0.0029 & 0.3956 & 0.9561 \\
\hline $\mathrm{RCA}$ & $\mathrm{M}$ & 129 & 4 & 3.5 & 0.025 & 0.0336 & 7.065 & 9.4954 \\
\hline LCA & $\mathrm{F}$ & 8.1 & 4.1 & 3.4 & 0.0009 & 0.0023 & 0.2534 & 0.5778 \\
\hline RCA & $\mathrm{F}$ & 123 & 3.7 & 3.3 & 0.0253 & 0.0342 & 6.3554 & 8.591 \\
\hline
\end{tabular}
approved parameters such as length and diameter of coronary branches, blood viscosity and its velocity.

Table 1- Clinically approved parameters to calculate blood flow rate

\begin{tabular}{|l|l|l|l|l|}
\hline Coronary Arteries & Sex & Diameter $(\mathrm{mm})$ & Length(mm) & $\begin{array}{l}\text { Blood Viscosity } \\
\left(10^{-3}\right)\end{array}$ \\
\hline LCA & $\mathrm{M}$ & $4.5 \pm .5$ & $9.7 \pm 4.3$ & $3.57 \pm 0.43$ \\
\hline RCA & $\mathrm{M}$ & $3.6 \pm .8$ & 124 to 140 & $3.57 \pm 0.43$ \\
\hline LCA & $\mathrm{F}$ & $4.1 \pm .8$ & $8.5 \pm 4.2$ & $3.30 \pm 0.20$ \\
\hline RCA & $\mathrm{F}$ & $3.2 \pm .9$ & 120 to 134 & $3.30 \pm 0.20$ \\
\hline
\end{tabular}

\subsection{Simulation Results of Healthy and Diseased Coronary Arteries}

Table 2. Comparison between resistance and blood flow of healthy and diseased coronary arteries 


\section{Measurement of Resistance:}
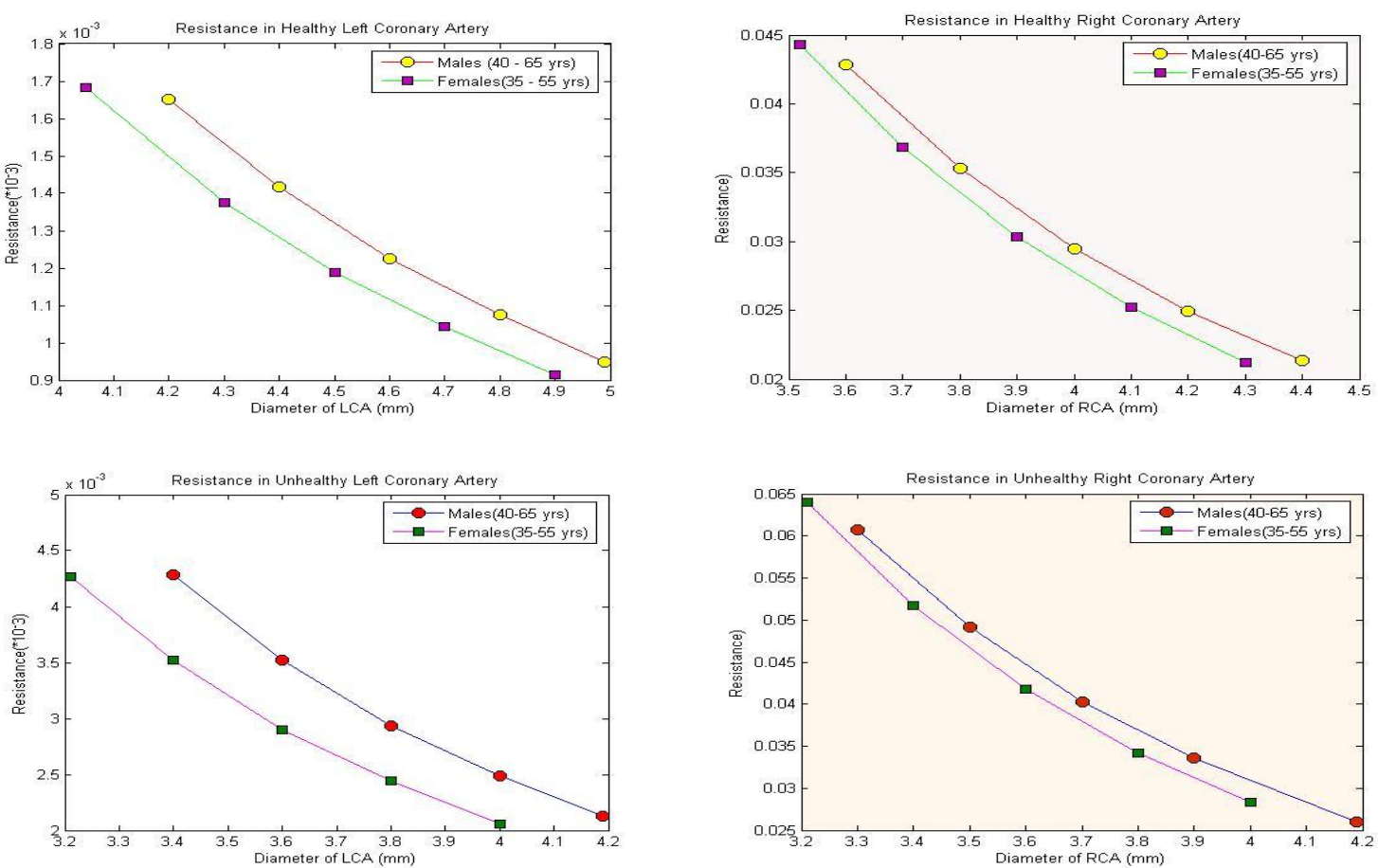

Fig 5: Resistance against diameter of healthy and diseased coronary arteries (from Eq. 13)

\section{Measurement of Blood Flow:}
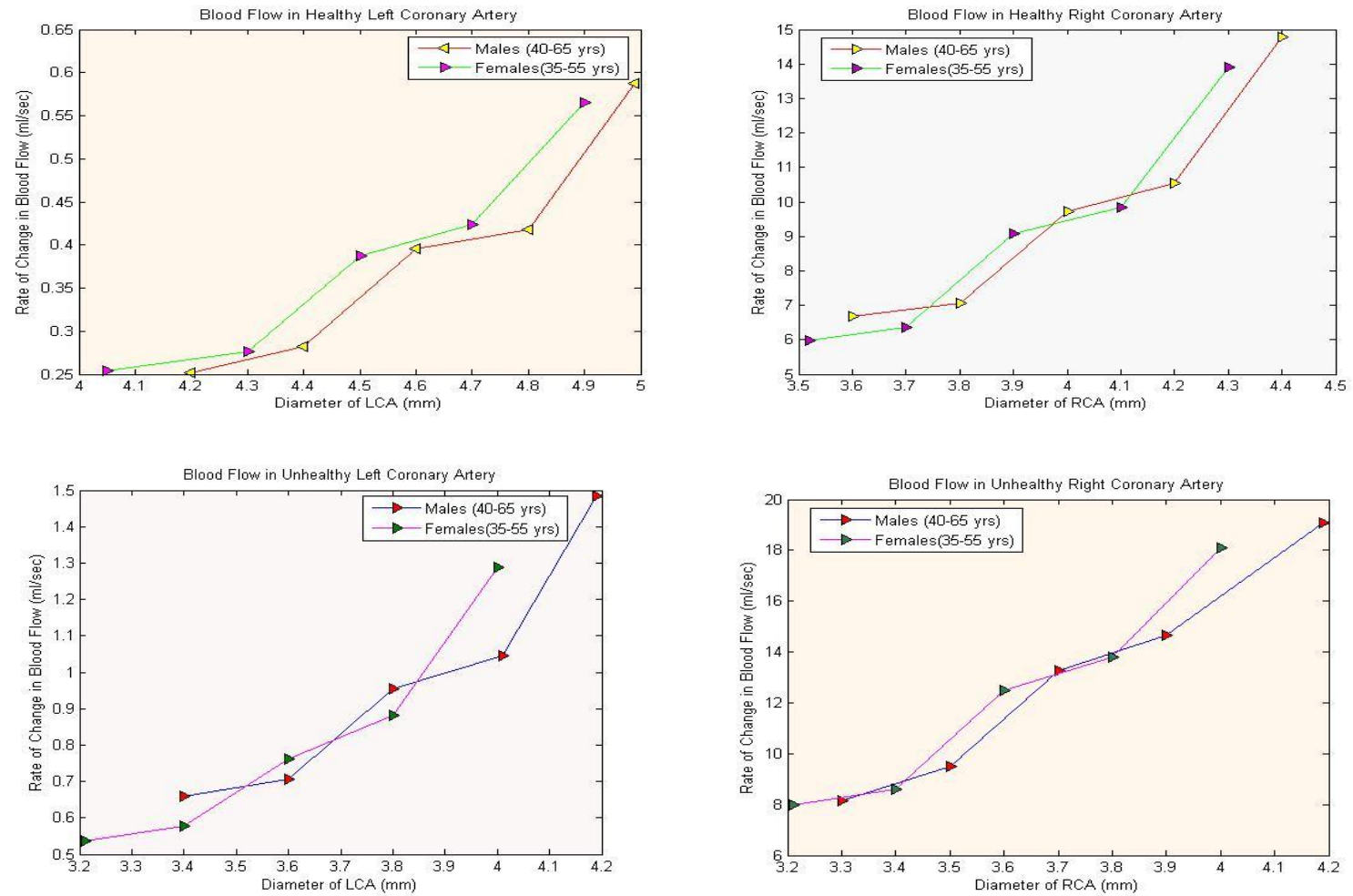

Fig 6: Blood flow against diameter of healthy and diseased coronary arteries (from Eq. 12) 


\section{Simulation of Wall Shear Stress:}

(a)
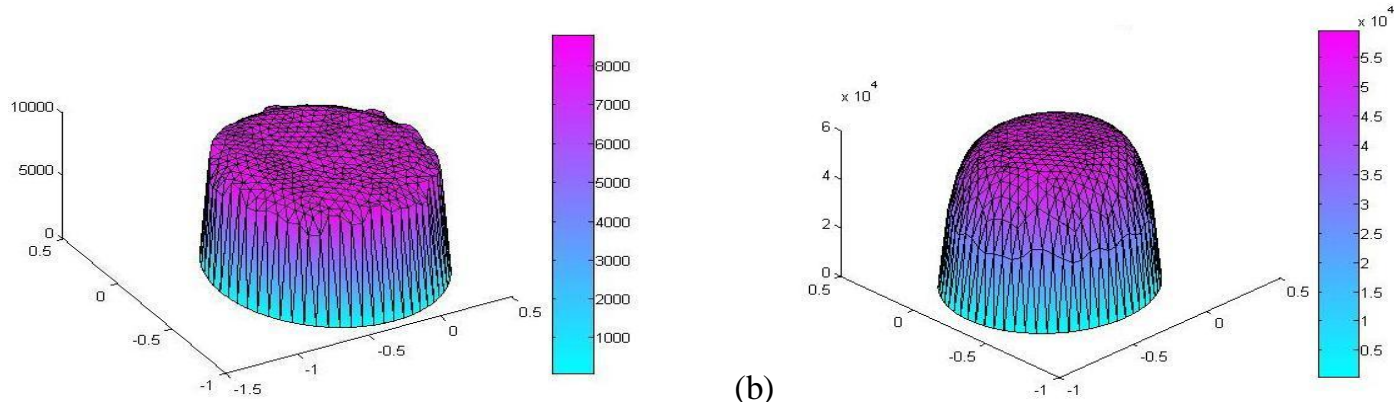

Fig 7: (a) (b) Simulation of wall shear stress rate in $2^{\text {nd }}$ order polynomial (using MATLAB PDE tool) of healthy and diseased coronary arteries respectively (from Eqs. $14 \sim 15$ )

\subsection{Cross Sectional Area Measurements and Degree of Stenosis Detection}

Variations in the area of artery can be a symbol of stenosis or blockage. Degree of stenosis can be quantified as follows which results to measure risk factors of heart attack.

$$
\% \text { Stenosis }=\frac{A_{N}-A_{B}}{A_{N}} \times 100
$$

where $A_{N}$ is area of normal artery and $A_{B}$ is effective or diseased area of artery

Table 3. Measurements of Cross Sectional Area and degree of Stenosis in selected Coronary Arteries

\begin{tabular}{|l|l|l|l|l|l|}
\hline Coronary Artery & Sex & $\begin{array}{l}\text { Area of artery } \\
\text { without blockage } \\
\left(\mathrm{in} \mathrm{m}^{2}\right)\end{array}$ & $\begin{array}{l}\text { Area of artery } \\
\text { due to blockage } \\
\left(\mathrm{in} \mathrm{m}^{2}\right)\end{array}$ & $\begin{array}{l}\text { \% Blockage } \\
\text { (Stenosis) }\end{array}$ & $\begin{array}{l}\text { CHD Risk } \\
\text { Factor }\end{array}$ \\
\hline LCA & $\mathrm{M}$ & 0.011 & 0.007 & $36.36 \%$ & Mild \\
\hline RCA & $\mathrm{M}$ & 0.010 & 0.005 & $50 \%$ & Severe \\
\hline LCA & $\mathrm{F}$ & 0.009 & 0.004 & $55.55 \%$ & Severe \\
\hline RCA & $\mathrm{F}$ & 0.005 & 0.004 & $20 \%$ & Initial \\
\hline
\end{tabular}

LCA-Left Coronary Artery, RCA-Right Coronary Artery, M-Male, F-Female, CHD-Coronary Heart Disease

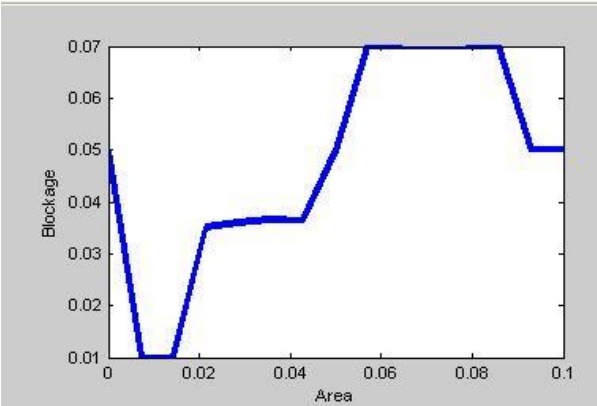

Fig 8: (a) Blockage corresponds to area

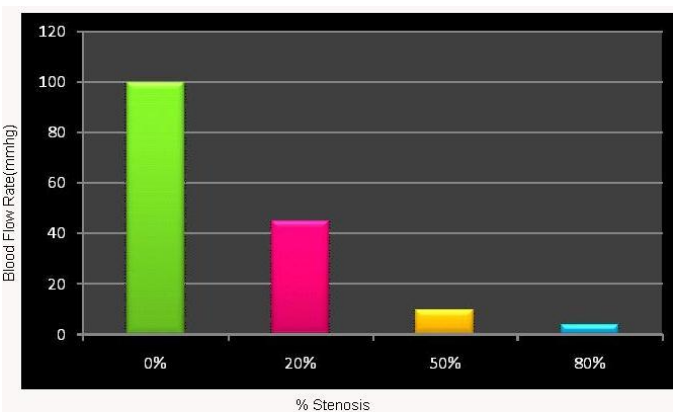

(b) \% Blockage corresponds to blood flow rate

\section{Conclusions}

This study has proposed a semi-automated approach for segmentation of coronary arteries as well as quantifies the degree of stenosis. Modeling of blood flow gives valuable results with observation that variations in cardiac vessels's diameter make vast changes in blood flow rate. Further, study needs improvement in area of computerized simulations for better findings

\section{Acknowledgement}

We would like to express our gratitude to Dr.N.Vikramaditya Yadav, Senior Inteventional Cardiologist, Klinikum Nurenbrg Hospital Germany, Dr. Manpreet Singh Bindra, Cardiologist, Cardio Life Care Ludhiana and last but not least the Team of Dr. Balabhai Nanavati Hospital, Mumbai for their help and guidance which helped out to carry this research work 


\section{References}

[1]. Oksuz Ilkay, Unay Devrim, Kadipasaoglu Kamuran (2012) A Hybrid Method for Coronary Artery Stenoses Detection and Quantification in CTA Images, funded project of Turkish Ministry of Science, Industry, and Technology (grant number 00706.STZ.2010-2)

[2]. Yang Guanyu, Kitslaar Pieter, Frenay Michel, Broersen Alexander, Boogers J.Mark, Bax J.Jreoen, Reiber.C.H Johan, Dijkstra Jouke (2011) Int J Cardiovasc Imaging (2012) 28:921-933,DOI 10.1007/s10554-011-9894-2

[3]. Corciova.C, Turnea.M, Corciova.F Analysis of Blood Flow in Coronary Arterial Tree Using Mathematical Models (2012), Proceedings of the 1st International Conference on Health Science and Biomedical Systems (HSBS '12) ISBN: 978-1-61804-097-8

[4]. Labadin.J, Ahmadi.A (2006) Mathematical Modeling of the Arterial Blood Flow Mathematical Modeling of the Arterial Blood Flow, Proceedings of the $2^{\text {nd }}$ IMT-GT Regional Conference on Mathematics, Statistics and Applications, Universiti Sains Malaysia, Penang

[5]. Fibich.G, Lanir.Y, Liron.N (1993) Mathematical model of blood flow in a coronary capillary, Am. J. Physiol. 265 (Heart Circ. Physiol. 34): H1829-H1840

[6]. Mukherjee Saurabh, Agrawal Supriya, Sahu Priyanka, Pruthi Jyotika (2012) Analysis of Heat Flow Produced by Specific Heating Modality on Selected Region in a body. Proceedings of ISCI-2012, Indian Institute of Technology, Bombay, p_id 66.

[7]. Mazinani Mahdi, Qanadli D.S, Hosseini Rahil, Ellis Tim, Dehmeshki Jamshid (2011) A 3D Approach for Extraction of the Coronary artery and Quantification of the Stenosis, World Academy of Science, Engineering and Technology 59

[8]. Frangi.F. Alejandro, Niessen.J. Wiro, Vincken.L. Koen, Viergever.A.Max (1998) Multiscale vessel enhancement filtering, Lecture Notes in Computer Science, vol.1496-Springer Verlag, Berlin Germany, pp 130-137

[9]. S.Milos, N.Amiya (2007) Direct ellipse fitting and measuring based on shape boundaries, Proceeding of the 2nd Pacific Rim conference on Advances in image and video technology 